\title{
Cystic Lymphangioma: an Uncommon Cause of Pediatric Abdominal Pain
}

\author{
Iván Fernandez-Vega ${ }^{1,2 *}$ \\ ${ }^{1}$ Service of Anatomic Pathology, Hospital Universitario de Araba-Txagorritxu, Spain \\ ${ }^{2}$ Service of Anatomic Pathology, Hospital Universitario Central de Asturias, Spain
}

*Corresponding author: Dr. Iván Fernández-Vega, PhD, MD, Service of Anatomic Pathology, Hospital Universitario Central de Asturias, Avenida de Roma s/n, E-33011, Oviedo, Asturias, Spain, Tel: 3494-500-7001, E-mail: ivan_fernandez_vega@hotmail.com

\begin{abstract}
Lymphangiomas are benign congenital malformations characterized by an abnormal dilatation and proliferation of lymphatic spaces, which are found predominantly in children. Symptomatic mesenteric cyst accounts for only 1 in 20,000 in acute pediatric admission. We describe hereby a case of a previously healthy 6-year-old girl who presented acute three-day pain localized over the right upper abdominal quadrant due to intestinal obstruction. A huge irregular and multi-lobulated mass measuring $17 \times 15$ $\mathrm{cm}$ and located in the mesentery was surgically resected. Pathological and immunohistochemical features of the lesion were consistent with cystic lymphangioma, supported by positive inmmunorreaction for D2-40 (podoplanin) in endothelial cells. Finally, she was discharged after a few days of hospitalization, and she currently is free of disease and symptoms.
\end{abstract}

\section{Main Text}

Lymphangiomas are benign congenital malformations characterized by an abnormal dilatation and proliferation of lymphatic spaces, which are found predominantly in children. They result from an embryological failure of the lymphatic system as consequence of absence of communication between small bowel lymphatic tissue and the main lymphatic vessels during fetal development result in blind cystic lymphatic spaces lined by endothelial layers. Lymphangiomas usually appear in head and neck regions and rarely in the mesentery or retro peritoneum [1]. Furthermore, they are frequently asymptomatic, but sometimes they may cause fatal complications such as volvulus, ischemia or intestinal obstruction requiring an urgent surgical intervention, that most of the time is both a therapeutic and diagnostic procedure [2]. In fact, published data demonstrated that abdominal lymphangiomas have a male preponderance and present more acutely in pediatric patients, whereas in adults, female patients predominate and the history is more chronic [3]. Specifically, symptomatic mesenteric cyst accounts for only 1 in 100,000 in acute adult and 1 in 20,000 in acute pediatric admission [4].

We describe hereby a case of a previously healthy 6 -year-old girl who presented acute three-day pain localized over the right upper abdominal quadrant. She did not mention nausea, vomiting or fever. On radiology, dilated loops of small bowel were pointed out and intestinal obstruction was confirmed by abdominal ultrasound. An emergency laparotomy was carried out. When surgeons were in the middle of the intervention, they surprisingly saw a diffuse peritonitis with free-intrabdominal milky fluid due to rupture of a cystic mass. Then, a $30 \mathrm{~cm}$ segment of small bowel was resected.

The surgical specimen showed a diffuse inflammatory appearance of the small bowel with a huge irregular and multi-lobulated mass measuring $17 \times 15 \mathrm{~cm}$. This mass was located mainly in the mesentery (Figure $1 \mathrm{~A})$. Cut section highlighted a multi-cystic lesion filled with abundant lacteous fluid (Figure 1B and Figure 1C).

Histologic examination of the mass revealed numerous dilated lymphatic spaces, sometimes filled with

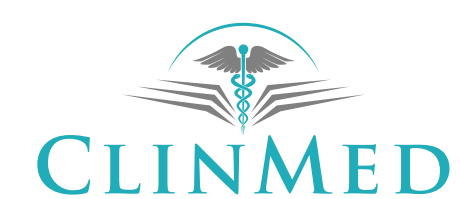

INTERNATIONAL LIBRARY 

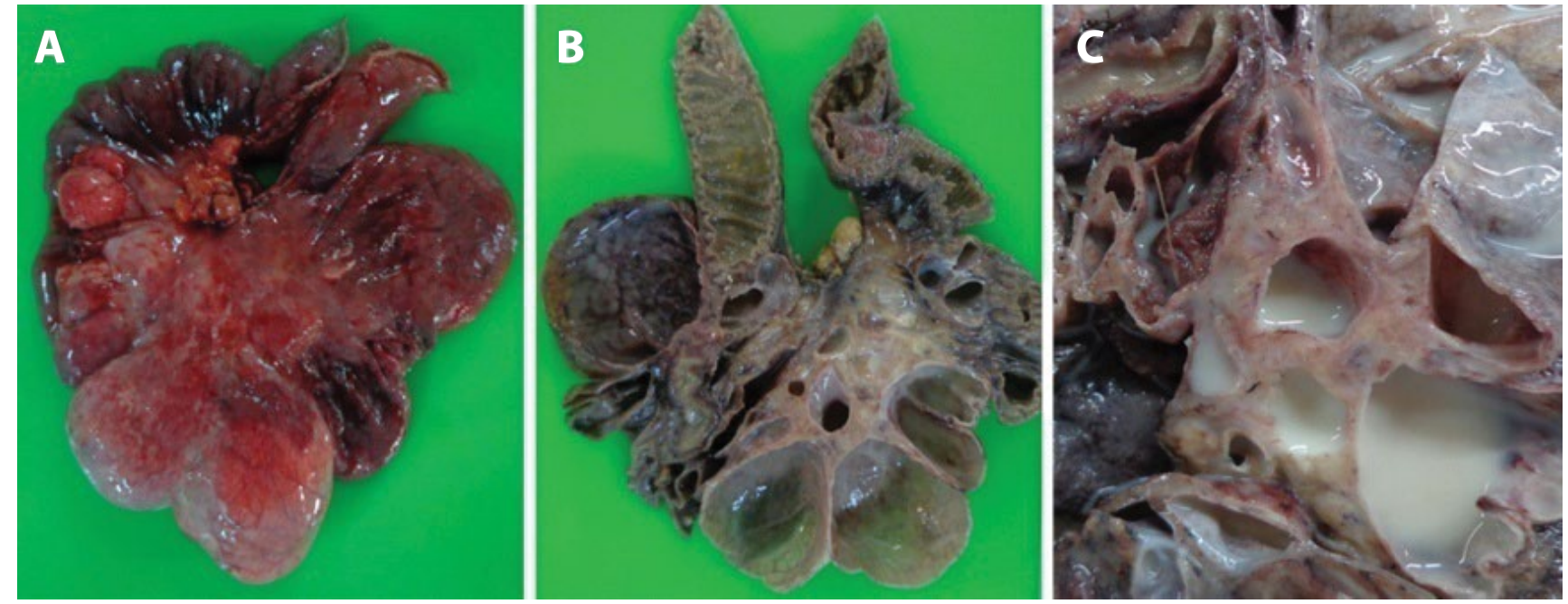

Figure 1: Gross pathology of a lobulated cystic yellowish pink mass that originated from the small-bowel mesentery with small bowel dilatation A) Fresh tissue immediately after surgical resection; B) The cut surface of the fixed tissue reveals thinwall multicystic spaces with thickened white appearance in some cystic walls. The small-bowel mucosa is unremarkable; C) Close up microphotography shows milky white fluid in the cysts.
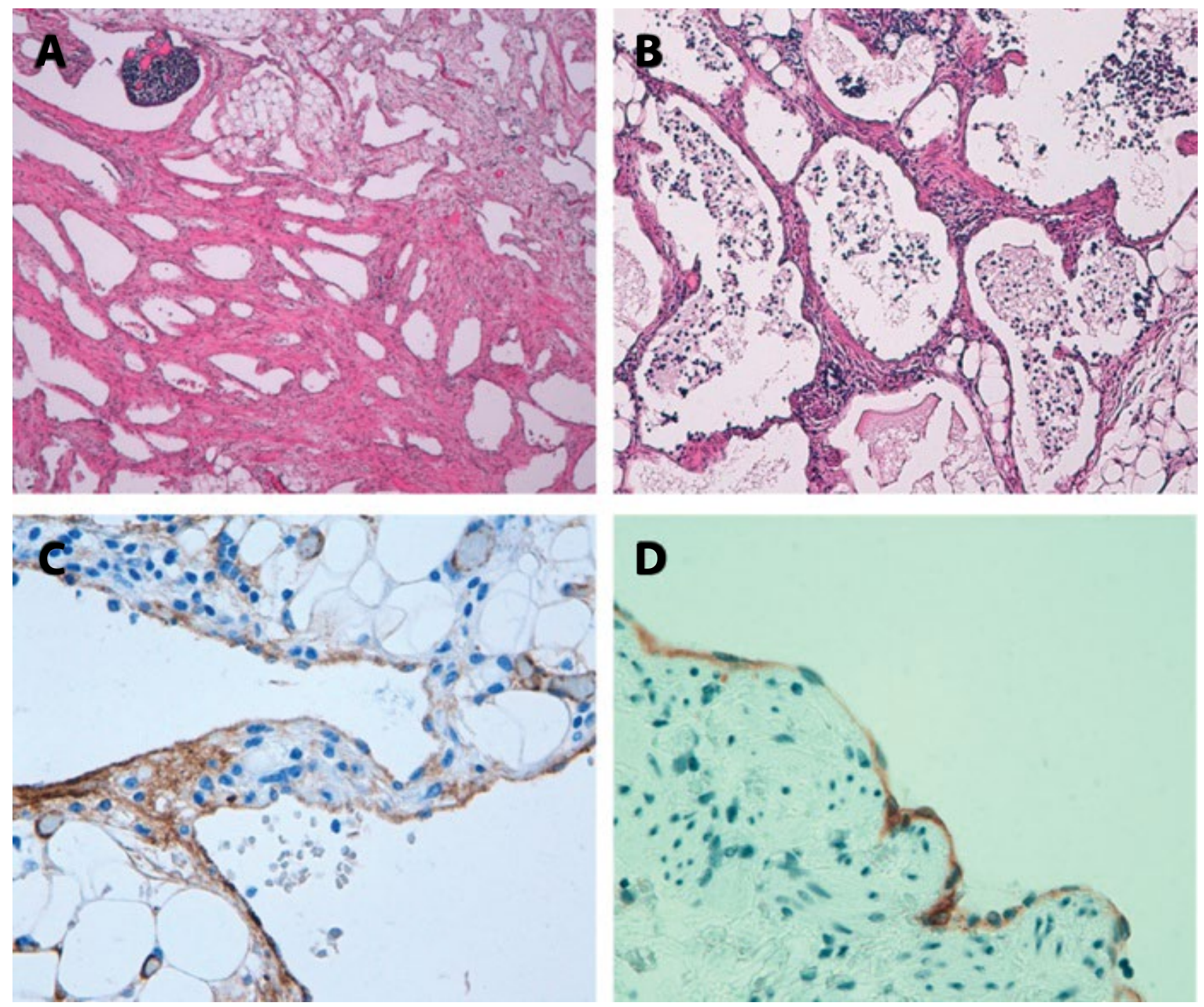

Figure 2: Histological features of the cystic tumor A) Cystic spaces with smooth muscle in their walls (H\&E, $x 100)$; $B)$ Lymphatic spaces in the stroma containing lymphoid cells and pale eosinophilic fluid, suggestive of lymph (H\&E, x200); C,D) Immunohistochemical study of the lining cells of the cystic wall shows strong cytoplasmic immunoreactivity for factor VIII-related antigen (C, x400) and D2-40 (D, x400). All immunostaining was performed by immunoperoxidase technique on paraffin-embedded tissue sections. 
lymph liquid and large numbers of foamy macrophages (Figure 2A and Figure 2B). The cystic walls were lined by non-atypical endothelial cells positively immune reactive for CD31, CD34, D2-40 (podoplanin) and factor VIII; negative for HMB45, CK5/6 and calretinin (Figure $2 \mathrm{C}$ and Figure 2D). The underlying stromal tissue showed some smooth muscle layers, collagen bundles, adipose tissue and occasional lymphoid aggregates. Neither mitotic figures nor necrotic areas were noted (Figure 2B). Taking all together the definitive histopathological diagnosis was of cystic lymphangioma of the small bowel mesentery [5].

From a pathological point of view, and taking into account some similarities between mesentery cystic lymphangiomas and other intrabdominal cystic tumors such as enteric duplication cyst and peritoneal cystic mesothelioma, a wider immunohistochemical study were performed [6]. Our tumoral lesion was completely negative for multy-cytokeratin (AE1/AE), calretinin and HMB45. However, it showed an intense positivity for vascular markers, especially for D2-40, a reliable lymphatic antibody [5].

Finally, the patient was discharged after a few days of hospitalization and she is free of disease and symptoms after 3 years of follow-up. No other congenital abnormalities were found in association.

\section{Funding Sources}

None.

\section{Conflict of Interest}

None.

\section{References}

1. Wani (2009) Mesenteric lymphangioma in adult: a case series with a review of the literature. Dig Dis Sci 54: 27582762.

2. Gimeno Aranguez M, Colomar Palmer P, Gonzalez Mediero I, Ollero Caprani JM (1996) The clinical and morphological aspects of childhood lymphangiomas: a review of 145 cases. An Esp Pediatr 45: 25-28.

3. Goh BK, Tan YM, Ong HS, Chui CH, Ooi LL, et al. (2005) Intra-abdominal and retroperitoneal lymphangiomas in pediatric and adult patients. World J Surg 29: 837-840.

4. de Perrot M, Brundler M, Totsch M, Mentha G, Morel P (2000) Mesenteric cysts. Toward less confusion? Dig Surg 17: 323-328

5. Rudno-Rudzinska J, Kielan W, Grzebieniak Z, Dziegiel P, Donizy P, et al. (2013) High density of peritumoral lymphatic vessels measured by D2-40/podoplanin and LYVE-1 expression in gastric cancer patients: an excellent prognostic indicator or a false friend? Gastric Cancer 16: 513-520.

6. De Bast Y, Hossey D, Boon L, Duttmann R, Theunis A, et al. (2006) Intra-abdominal lymphatic malformation. Acta Gastroenterol Belg 69: 209-212. 